\title{
Évaluation du risque toxicologique des OGM
}

Oléagineux, Corps Gras, Lipides. Volume 11, Numéro 4, 241-5, JUILLET-OCTOBRE 2004, OGM

\author{
Auteur(s) : Gérard Pascal
}

INRA. Direction scientifique Nutrition humaine et sécurité des aliments, 147, rue de l'Université, 75338 Paris cedex 07.

Summary : Two French scientific committees, the committee of biomolecular engineering (CGB) and the committee on pesticide toxicity (CT), decided to hold a common conference on the 27th and 28th September 2002. They have indeed each one to evaluate certain specific aspects of the risks related to the use of genetically modified plants (GMP), in particular herbicide tolerant, or resistant to insects because of secretion of their own insecticide. The participants in the conference compared their methodologies of evaluation of risks for human and animal health, underlined their strong points and their weaknesses. Rare specificities for the evaluation of the GMP were identified compared to food risk assessment. New methods, based on progress of genomics and post-genomics should make it possible to improve the sensitivity of detection of effects of low amplitude due to exposure not only to the GMP, but also to novel foods and new technologies.

Keywords : genetically modified plants, risks for human and animal health, methodologies of evaluation of risks

\section{ARTICLE}

Le texte présenté ici est le compte rendu d'un colloque organisé conjointement par la Commission du génie biomoléculaire (CGB) et la Commission d'étude de la toxicité des produits antiparasitaires à usage agricole (CT), les 27 et 28 septembre 2002 au Sénat.

Au cours des dernières années, la Commission du génie biomoléculaire (CGB) a examiné de nombreux dossiers de demande de dissémination dans l'environnement, voire de mise sur le marché, de plantes génétiquement modifiées résistantes à des herbicides ou de produits alimentaires issus de ces plantes. L'évaluation de leur sécurité sanitaire suppose de prendre en compte une situation nouvelle, c'est-à-dire les conséquences de l'application sur les plantes d'une substance qui ne l'était pas auparavant puisque les plantes traditionnelles de l'espèce considérée ne résistaient pas à la substance et auraient donc été détruites.

Si la question était régulièrement posée au sein de la CGB, sa réponse ne revenait pas à cette commission puisque l'évaluation de la sécurité d'emploi des produits phytosanitaires est réglementairement du ressort de la Commission d'étude de la toxicité des produits antiparasitaires à usage agricole et des produits assimilés, des matières fertilisantes et des supports de culture (CT). La situation nouvelle qui résulte de la résistance de plantes à un herbicide impose évidemment un examen par la CT. Cependant, l'examen de façon indépendante, par deux commissions différentes, d'aspects complémentaires d'un même dossier d'évaluation de la sécurité sanitaire d'une plante 
génétiquement modifiée ne permet pas à leurs membres d'avoir une vision intégrée et laisse subsister des interrogations quant au jugement final. Une amélioration sensible de cette situation consistait a minima à organiser un échange de vues entre ces membres, de façon à avoir une vision partagée des méthodes d'évaluation de risque appliquées par les uns et les autres, de mettre en lumière les difficultés, les spécificités et les convergences des différentes situations rencontrées. Les présidents des deux commissions ont donc décidé d'organiser un colloque commun réunissant les membres des deux commissions, ainsi qu'un certain nombre d'invités impliqués dans des évaluations de risques dans d'autres domaines, comme celui des produits chimiques ou des médicaments.

Ce colloque s'est tenu les 27 et 28 septembre 2002 au Sénat où le Sénateur Jean Bizet, qui y présidait alors la Mission d'information sur les OGM, avait bien voulu nous inviter.

Le colloque avait été conçu en trois parties :

- la première consistait à décrire les différentes étapes de l'évaluation des risques des produits phytosanitaires en l'élargissant à l'évaluation du risque allergène en général ;

- la deuxième était consacrée à la description des approches actuellement utilisées aux plans national et européen dans le cas des OGM et de leurs traitements phytosanitaires, objets d'un examen par les commissions ad hoc ;

- la troisième avait comme objectif, à partir des points faibles identifiés lors des discussions précédentes, dans le but d'améliorer la situation actuelle, de passer en revue les nouvelles approches d'évaluation de risque rendues possibles grâce aux progrès fulgurants de la biologie. Les points forts et les incertitudes liés à ces méthodologies applicables certes aux OGM, mais aussi aux perturbateurs endocriniens par exemple ou aux aliments en général, ont été passés en revue. L'intégralité des présentations et des discussions de ce colloque est publiée par INRA-Editions, sous forme électronique.

\section{Les méthodologies classiques d'appréciation des risques : l'exemple des risques phytosanitaires}

Daniel Marzin, le président de la CT, a décrit dans le détail les étapes de l'appréciation du risque d'usage des produits phytosanitaires : risques liés à la matière active (propriétés physico-chimiques, méthodes analytiques, métabolisme et toxico-cinétique, différents aspects de l'évaluation toxicologique), détermination des doses de référence et, enfin, évaluation des risques pour l'homme de la préparation qui sera utilisée sur le terrain.

La caractérisation du risque pour le consommateur suppose la connaissance de l'exposition de l'homme, connaissance qui exige à la fois la détermination des résidus dans les produits alimentaires et l'évaluation du niveau de consommation de ces produits. Détermination des résidus et évaluation des consommations et, en définitive, des expositions, ont été successivement abordés par Bernard Declercq (DGCCRF) et Jean-Charles Leblanc (INRA) [1]. La discussion qui a suivi ces présentations a porté sur l'importance qu'il convenait d'attacher aux expositions aiguës (en particulier des applicateurs) et à celles des enfants.

Enfin, Bernard David (Institut Pasteur), spécialiste de l'allergie, a souligné que l'allergie alimentaire ne 
représentait que $2 \%$ des allergies chez l'adulte et qu'il ne fallait pas confondre allergie et sensibilisation et encore moins toxicité, infection, irritation, bref toute réaction inflammatoire pouvant suggérer une allergie [2]. La gravité des rares chocs anaphylactiques dus à l'alimentation impose cependant de ne pas construire de transgènes avec des allergènes répertoriés comme tels dans les banques de données et surtout d'avertir les consommateurs sensibles de la composition des aliments complexes par l'étiquetage, afin qu'ils puissent éviter la consommation d'aliments qui leur sont nocifs alors qu'ils sont anodins pour la plupart des individus [3]. Au total, il apparaît d'une façon générale que la prédiction de l'allergénicité d'une molécule inconnue est quasiment impossible, si on ne peut la comparer à un allergène déjà identifié et répertorié. L'expérimentation animale n'est pas aujourd'hui en mesure de répondre, car toute protéine peut, en fonction des conditions de stimulation et de la génétique du receveur, créer soit un état d'hypersensibilité (IgE), soit une réponse immunitaire (IgG).

Une table ronde a ensuite abordé les approches, spécificités et difficultés rencontrées dans d'autres domaines que le phytosanitaire : Claire Beausoleil (INRS) et Claude Alabouvette (INRA) ont évoqué successivement les produits chimiques industriels et les biopesticides et plus spécifiquement les micro-organismes employés en lutte biologique. Le cas des produits chimiques est théoriquement proche de celui des produits phytosanitaires; la méthodologie d'appréciation des risques devrait être identique, cependant, en pratique, les informations disponibles sont souvent bien plus pauvres. Les voies d'exposition professionnelle sont le plus souvent l'inhalation ou le contact cutané à la différence de l'ingestion dans le domaine alimentaire. Une connaissance de l'ensemble des sources d'exposition fait souvent défaut.

L'appréciation des risques des micro-organismes employés en lutte biologique présente quelques spécificités en particulier en matière de caractérisation de la souche et d'impureté des préparations, par exemple en matière de toxines ou d'antibiotiques.

\section{Les approches utilisées dans le cas des OGM}

Dans un premier temps, Louis-Aymé Aumaitre (INRA) a dressé un panorama complet des tests réalisés principalement chez l'animal de ferme [4]. En effet, beaucoup des tests toxicologiques réalisés chez l'animal de laboratoire ne sont pas accessibles en raison de la confidentialité des données, mêmes si les résultats de certains d'entre eux ont été publiés dans la littérature scientifique. Les données disponibles, nombreuses contrairement à ce que certains prétendent, concernent essentiellement le maïs et le soja résistants aux herbicides ou à la pyrale pour le maïs, mais aussi le colza ou la betterave dans une moindre mesure. Les espèces animales concernées sont le poulet, le porc, le mouton et les bovins (taurillons, vaches laitières). Les tests réalisés ne sont pas des tests toxicologiques, mais des tests d'alimentarité et de tolérance chez les espèces animales auxquelles sont destinés la plupart des OGM testés.

Aumaitre a conclu que les nouvelles plantes modifiées testées sont " équivalentes en substance ", à la fois en termes de composition chimique (nutriments et facteurs anti-nutritionnels), de digestibilité, de valeur alimentaire et de performances des animaux, lorsqu'on les compare avec les données observées sur des animaux nourris avec des plantes parentales. Les tests in vivo sur animaux de ferme sont à recommander de façon à prévenir tout risque lié à la présence fortuite de facteurs anti- 
nutritionnels ou toxiques connus ou inconnus. L'absence, chez les animaux ou leurs produits, d'ADN ou de fragment d'ADN issus de la construction génique avait été confirmée à l'époque dans onze expérimentations sur onze publiées, de même que celle d'ADN recombinant dans les bactéries du rumen, lors de la mis en présence d'aliment à base de colza modifié.

Hubert Noteborn (Dutch Food and Non Food Authority) a ensuite présenté en détail le concept d'équivalence en substance et la méthodologie de sa démonstration. Le premier à avoir testé avant 1995 (date de sa première publication sur le sujet) une tomate-Bt, il a été depuis très fortement impliqué dans plusieurs programmes européens de recherche du cinquième PCRD, en particulier Gmocare et Entransfood dont l'un des objectifs consiste à améliorer l'évaluation de cette équivalence en substance et à en accroître la signification dans le processus d'appréciation du risque sanitaire des OGM [5]. Ces progrès reposent en partie sur la mise en œuvre des méthodes en " omique ", transcriptomique, protéomique, métabolomique. H. Noteborn a cependant souligné les difficultés d'interprétation des données ainsi obtenues et donc de leur application en routine pour le moment, mais aussi la possibilité de mettre ainsi en évidence des effets non intentionnels des transformations génétiques.

René Scalla a très précisément détaillé les mécanismes qui rendent les plantes transgéniques résistantes aux herbicides (glyphosate, glufosinate ou phosphinotricine, bromoxynil et thiazopyr) et quel est alors le devenir des herbicides appliqués, l'accumulation de résidus et les modifications du métabolisme des plantes ainsi provoquées. Cette présentation permet de mieux appréhender les questions spécifiques qui doivent être abordées pour apprécier le risque sanitaire de ces OGM et de leurs traitements phytosanitaires [6].

L'objectif de la table ronde qui a suivi ces trois présentations, à laquelle ont participé lb Knudsen (Institue of Food Safety and Nutrition, Danemark), Gilles-Eric Séralini (Université de Caen) et moimême, Gérard Pascal (INRA), consistait à se livrer à une analyse critique des méthodes actuelles d'évaluation des OGM.

Ib Knudsen a évoqué une expérimentation en cours, réalisée dans le cadre du projet européen Entransfood déjà cité par $\mathrm{H}$. Noteborn, et dans laquelle des constructions codant pour des lectines toxiques ont été introduites dans des riz. La toxicité de ces lectines, exprimées dans le riz, est comparée à celle des mêmes lectines ajoutées à un riz non transgénique, afin de mettre en évidence une éventuelle conséquence non intentionnelle de la transformation génétique. Il a fallu introduire des gènes codant pour des produits manifestement toxiques pour que les tests toxicologiques classiques utilisés par ailleurs, permettent d'enregistrer des signes de toxicité qu'ils n'ont pas permis de révéler jusqu'à présent avec les OGM proposés pour une mise sur le marché.

Gilles-Eric Séralini, qui s'est félicité de l'organisation d'un tel colloque, s'est livré à une analyse critique des pratiques actuelles. II a regretté l'introduction de séquences virales qui pourraient favoriser les recombinaisons génétiques et la susceptibilité des plantes aux agressions virales. Soulignant la légitimité des inquiétudes du public vis-à-vis des OGM, il a déploré le faible nombre d'études à 90 jours chez l'animal de laboratoire et l'absence d'études à long terme. Il a regretté aussi l'absence d'évaluation des risques endocriniens et immunitaires et tenant compte des différentes formulations des préparations phytosanitaires. II a plaidé pour l'application aux OGM des méthodologies utilisées pour l'appréciation des risques de ces produits. 
J'ai personnellement voulu souligner les différences, fondamentales à mon sens, entre l'appréciation des risques de molécules chimiquement définies, conçues pour avoir un effetpharmacologique ou toxique comme les médicaments ou les produits de traitement des plantes et pour lesquelles l'exposition de l'homme est faible, et l'appréciation des risques liés à la consommation d'un aliment, de composition extrêmement complexe, qui peut constituer une part importante de l'ingéré alimentaire quotidien. Dans ce dernier cas, ce n'est qu'une action discrète et à long terme sur l'organisme consommateur que l'on recherchera, la toxicité aiguë étant facilement détectable. Des situations intermédiaires se rencontrent dans le cas de certains contaminants toxiques, également à exposition quantitativement faible et de substances par définition inertes au plan biologique, comme les additifs alimentaires, mais dont l'exposition est aussi réduite.

Dans le premier cas, les méthodes de la toxicologie classique sont bien adaptées; on expose les animaux de laboratoire à de très fortes doses, sans commune mesure avec les expositions réelles de I'homme, et on définit des doses journalières acceptables (DJA) en appliquant un facteur de sécurité important, en général de 100, aux doses maximales sans effet observable chez l'animal. Cette méthodologie est parfaitement applicable à l'évaluation des protéines nouvellement exprimées à très faible concentration dans les OGM, mais elle est inadaptée aux aliments pour lesquels il est pratiquement impossible de forcer la dose et donc de mettre en évidence des effets par ailleurs de faible amplitude. Elle ne convient donc pas, car insuffisamment sensible, pour mettre en évidence les effets inattendus, imprévisibles, liés à l'introduction d'une nouvelle construction génétique dans un génome. Elle serait seulement capable de détecter des effets réellement toxiques, et dans ce cas, des études subchroniques suffisent pour écarter de la consommation animale ou humaine des produits manifestement dangereux.

Ces différences dans l'appréciation des risques entre aliments ou macrocomposants des aliments et donc des OGM et molécules à faible exposition ont été soulignées dans un travail collectif publié à la suite d'une action concertée du cinquième PCRD européen, FOSIE [7].

Seule la mise au point de nouvelles approches plus sensibles permettrait de répondre à des questions, pour certaines légitimes, mais non spécifiques des OGM, venant des consommateurs.

La discussion générale qui a suivi a montré combien les points de vue réciproques étaient éloignés et combien il était difficile d'admettre que ce qui fonctionne bien dans un cas peut être inadapté dans un autre.

\section{Développements récents et approches toxicologiques nouvelles}

Cette troisième partie avait pour objectif de montrer, à partir de différentes préoccupations toxicologiques, quelles nouvelles approches pouvaient être explorées et éventuellement appliquées à l'appréciation du risque sanitaire des OGM. Françoise Paris (CHU Lapeyronie, Montpellier) et Mohamed Benhamed (Inserm U 407) ont présenté les modèles qu'ils utilisent pour élucider la nature des perturbateurs ou interrupteurs hormonaux et mieux comprendre leurs mécanismes d'action. Des produits phytosanitaires sont soupçonnés d'avoir de telles actions. Françoise Paris, en utilisant des modèles de cellules d'origine humaine ou de hamster transfectées, évalue les activités œstrogénique ou androgénique de divers contaminants de l'environnement. II s'agit d'une première approche qui doit être développée de façon à pouvoir tester des sérums humains en routine, sur des groupes de sujets soupçonnés d'avoir été exposés à des perturbateurs hormonaux et/ou présentant des 
pathologies pubertaires et pré-pubertaires. Mohamed Benahmed utilise lui un modèle animal de rat traité in utero, à différents stades de croissance embryonnaire, ou à l'âge adulte, avec le flutamide, molécule dont le dérivé actif se lie aux récepteurs des androgènes et empêche de ce fait l'activité de la testostérone. Ce modèle se révèle très intéressant puisque l'exposition intra-utérine des animaux au flutamide induit une hypospermatogenèse, malgré un bilan hormonal qui paraît tout à fait normal. Cet effet est dû à un processus chronique de mort cellulaire lié à une augmentation de l'expression et de l'activation de gènes des caspases 3 et 6 impliquées dans la mort cellulaire. Ce processus est irréversible lorsque l'exposition a lieu pendant la vie intra-utérine et réversible lorsqu'elle advient à l'âge adulte. Ce modèle peut constituer l'un des outils qui permettront de mieux connaître les mécanismes d'action des perturbateurs hormonaux par l'identification des gènes hormono-dépendants (par techniques en « ique "), l'élucidation du rôle de ces gènes d'intérêt (par transgénèse, transfection, électroporation...), en tenant compte des polymorphismes. Cette approche de génomique a constitué une bonne introduction à la présentation de Xavier Gidrol (Inra/CEA) sur l'utilisation des techniques de transcriptomique et protéomique dans une optique de " toxicogénomique » [8]. Responsable au CEA d'une unité préparant des puces ADN, il développe des programmes de recherche personnels en appliquant ces techniques par exemple à l'étude des effets du Cadmium (Cd) sur la levure. II a pu ainsi montrer que le $\mathrm{Cd}$ provoque dans la cellule une réorientation du métabolisme du soufre afin de permettre son utilisation prioritaire pour l'élimination du Cd. L'approche toxicogénomique permet d'obtenir des signatures moléculaires pour une famille de toxiques, c'est-à-dire des modifications d'expression de gènes qui peuvent intervenir assez précocement en réponse à l'exposition aux toxiques. Des bases de données d'expression sont en cours de création dans le monde, création qui demande un effort collectif. On pourra alors diagnostiquer l'exposition accidentelle d'un individu à un toxique, analyser les signatures moléculaires de contaminants xénobiotiques quels qu'ils soient et, potentiellement, examiner le polymorphisme de la réponse individuelle à un toxique. La technique de préparation de puces progresse rapidement et, grâce à des collaborations internationales, des puces pan-génomiques de plusieurs espèces constitueront des outils extrêmement puissants qui généreront des quantités énormes de données qu'il faudra être capable de traiter et surtout d'interpréter avec l'aide des bioinformaticiens.

Les techniques de métabolomique et de signatures biologiques mises en œuvre par Alain Paris [9], constituent un complément à celles décrites par Xavier Gidrol. Elles font appel à des méthodes de spectroscopie (de masse et RMN). Elles peuvent s'appliquer à des plantes OGM aussi bien qu'à des échantillons biologiques (sang, urines...) d'animaux qui ont consommé des OGM ou leur produits. C'est l'un des outils qui permet une estimation fine de l'équivalence en substance, car capable de mettre en évidence des différences de métabolisme d'une plante. Comme dans le cas des techniques de génomique, le véritable défi va consister non pas à mettre des différences en évidence, ceci sera très facile, mais à interpréter la signification de ces différences; adaptation physiologique à un environnement changeant reflétant la flexibilité des réseaux métaboliques ou manifestation d'un changement plus profond pouvant avoir des conséquence toxiques pour les organismes consommateurs. Les modifications enregistrées chez l'animal consommateur, présenteront les mêmes difficultés d'interprétation. Là encore, il faudra créer des référentiels avant d'imaginer appliquer ces techniques plus largement. Elles ont cependant déjà fait leurs preuves pour identifier, par exemple, les traitements hormonaux frauduleux dont certains bovins pourraient être l'objet et mettre en évidence les modifications métaboliques entraînées par ces traitements [10]. La dernière présentation de cette troisième partie, par Marc Fellous (Inserm), a concerné un travail 
réalisé en collaboration avec des chercheurs de la faculté de médecine de Cochin-Port-Royal et de I'Inra, sur les effets de la toxine Cry 1Ab exprimée dans certains OGM résistants à la pyrale du maïs, sur le mouvement des spermatozoïdes. Ce travail in vitro a montré de façon très claire l'énorme variabilité de réponse, d'une part d'un éjaculat à l'autre chez un même sujet et, d'autre part, entre spermes de différents donneurs. II n'a en définitive pas mis en évidence d'effet significatif de la toxine Cry $1 \mathrm{Ab}$ sur la mobilité des spermatozoïdes humains.

\section{Conclusions}

Une discussion générale fournie a suivi les présentations de la troisième partie du colloque et a repris certaines des questions abordées au cours des deux premières. J'en donnerai ci-après une analyse personnelle qui ne prétend pas à l'objectivité, mais qui correspondra à une perception personnelle des points de vue exprimés et de la distance qui sépare parfois ces points de vue. L'un des points qui a été je crois l'objet d'un consensus, consiste à considérer qu'il convient d'aborder l'appréciation de la sécurité sanitaire des OGM au cas par cas, et d'adapter les approches à chaque espèce végétale et à chaque construction, après avoir identifié les questions spécifiques posées par chaque transformation génétique. Une autre impression générale très forte, c'est la difficulté des uns et des autres, industriels des biotechnologies, toxicologues impliqués dans des domaines spécifiques, adversaires militants des OGM, à reconnaître que les techniques actuellement appliquées ne permettent pas de répondre à toutes les questions, mais que leur complémentarité apporte un niveau de sécurité au moins équivalent à celui des aliments dits courants, voire "naturels ", dont la sécurité n'a jamais été évaluée par quelque méthode que ce soit, mais dont on dit qu'ils ont une histoire d'utilisation sûre (history of safe use des Anglos-Saxons). Les aliments exotiques de plus en plus nombreux qui nous sont proposés ne bénéficient même pas de ce qualificatif.

Il est fondamental pour éviter un dialogue de sourds de bien préciser les limites des différentes approches :

- Possibilité d'utiliser les approches de toxicologie traditionnelle pour évaluer les produits des gènes d'intérêt et marqueurs, ou les résidus de pesticides ou leurs métabolites, pour lesquels l'exposition du consommateur animal ou humain est faible, la durée de ces tests (28 ou 90 jours) pouvant encore se discuter dans le cas des produits des gènes. - Utilisation de ces tests, en l'occurrence subchroniques à 90 jours (une majorité se dégage clairement pour cette durée), réalisés avec la plante entière ou ses produits. Ces tests ne seront capables que de mettre en évidence des effets toxiques marqués que des tests à 28 jours sont déjà capables de révéler (exemple du colza GT73). Sur la base de mon expérience dans le domaine des additifs alimentaires, molécules conçues comme sans effets biologiques a priori, de même que les OGM, je ne crois pas un instant que ces tests, même pratiqués à long terme ( 2 ans chez les rongeurs de laboratoire), soient capables de détecter des effets discrets, de faible amplitude, si on ne peut pas "forcer la dose " ("c'est la dose qui fait le poison! »), ce qui est le cas avec un aliment. De plus, comme les OGM ne sont pas à l'évidence des produits très toxiques, les données obtenues lors des essais de toxicologie classiques sont extrêmement difficiles à interpréter. Il est parfois délicat de faire la part entre les variations biologiques "normales » entre animaux d'expérience et un effet ayant une signification toxicologique, dû à la consommation de l'OGM. Un exemple récent est celui du maïs MON863 pour lequel l'interprétation des résultats de l'essai à 90 jours a été l'objet de divergences 
entre différents comités d'experts (CGB, Afssa et Efsa). Il est alors difficile d'expliquer dans la presse quotidienne, avec les nuances nécessaires, sans que les médias présentent les positions des scientifiques comme en opposition tranchée, les raisons qui peuvent motiver de telles divergences, soit parce que les journalistes ajoutent aux propos de ces scientifiques une touche personnelle, soit parce que les titres des articles s'écartent sensiblement de leur contenu [11, 12]. De telles divergences n'existent pas lorsque l'on se trouve face à un effet indiscutable, comme dans le cas de molécules actives à effet pharmacologique comme les médicaments, ou toxique comme les produits phytosanitaires.

- Utilisation de tests chez des animaux de ferme, bien souvent destinataires des OGM, pour en apprécier l'acceptabilité et l'alimentarité. II ne s'agit pas d'une évaluation toxicologique, mais ces tests permettraient de détecter des effets marqués, inattendus, imprévus, de l'insertion de la nouvelle construction génétique (ce qui n'a pas été le cas jusqu'à présent), surtout lorsqu'ils sont pratiqués sur des animaux à vitesse de croissance extrêmement rapide comme le poulet entre 1 et 42 jours.

Ces différentes déterminations interviennent après des comparaisons phénotypiques de la plante OGM avec la plante parentale. L'aspect, les caractéristiques agronomiques, les propriétés de résistance aux pestes, la composition en macro- et micronutriments et en substances antinutritionnelles ou toxiques sont comparés. Ces comparaisons participent à l'établissement de l'équivalence en substance. Ce concept souffre certes de critiques et le colloque en a bien démontré les points faibles, mais il peut être amélioré, en particulier lorsque nous disposerons de banques de données de composition des végétaux permettant d'apprécier l'importance des facteurs variétaux, agronomiques, climatiques, de variation. Ce concept devrait à mon sens être étendu pour juger de la sécurité des aliments dits nouveaux en Europe ou des produits issus de la sélection traditionnelle. Ce colloque m'a conforté dans l'impression qui était la mienne, selon laquelle l'évaluation actuelle des OGM apportait au consommateur une sécurité au moins équivalente à celle de ses aliments courants, voire supérieure. Je n'ai trouvé aucun argument qui puisse me faire considérer que tous les aliments ne devraient pas être l'objet du même questionnement que les OGM. J'ai acquis la conviction que les méthodes de la toxicologie classique, qui ont évité jusqu'à présent des catastrophes sanitaires, en matière d'additifs alimentaires par exemple, ne permettent pas de mettre en évidence des effets discrets d'un aliment ou d'un macroconstituant des aliments et que seules de nouvelles approches, telles que celles présentées en troisième partie du colloque, auront une sensibilité suffisante pour progresser encore dans l'assurance de la sécurité sanitaire de notre alimentation; de notre alimentation en général et pas seulement des OGM qui ne posent que bien peu de questions spécifiques, pas même celle de l'allergénicité. Je ne suis cependant pas certain d'avoir été seulement entendu; je n'avais bien sûr pas l'illusion d'être suivi. La sensibilité de ces nouvelles approches a été parfaitement démontrée. Reste à savoir gérer et surtout interpréter la montagne de données qu'elles vont générer; il s'agit du défi auquel se trouvent confrontées les équipes engagées dans ces voies. II s'agit non seulement d'un saut méthodologique, mais aussi conceptuel et les discussions qui ont eu lieu au cours de ce colloque ont bien montré qu'il existera toujours une lutte entre " les anciens et les modernes ". Mais c'est bien ce qui fait l'attrait de la recherche! Enfin, il est clairement apparu la nécessité a minima d'une information réciproque entre la $\mathrm{CGB}$ et la $\mathrm{CT}$, pour tout $\mathrm{OGM}$ résistant aux herbicides ou à certains insectes par sécrétion de pesticides. Il s'agit de situations nouvelles qui doivent être évaluées pour ce qui concerne la sécurité sanitaire des utilisateurs et consommateurs, selon les canons actuels de l'évaluation au sein de la CT. La CGB doit être informée des termes de la saisine de la CT et pouvoir 
lui communiquer les conclusions et questions résultant de l'examen des dossiers qui lui sont soumis. La multiplicité des commissions traitant des différents aspects d'un même dossier, ne participe pas à une vision intégrée des questions de sécurité qui sont l'objet d'une exigence accrue des citoyens, dans un contexte où la science devient de plus en plus pointue et spécialisée et ne facilite pas par elle-même cette intégration.

\section{RÉFÉRENCES}

1. LEBLANC JC, MALMAURET L, GUERIN T, BORDET F, BOURSIER B, VERGER P. Estimation of the dietary intake of pesticide residues, lead, cadmium, arsenic and radionuclides in France. Food additives and Contaminants $2000 ; 17: 925-33$.

2. DAVID B. Allergie immédiate, allergie retardée. Pour la science octobre 2000, dossier hors série : les défenses de l'organisme : 74-8.

3. EBNER C. Genetically Modified Food : A Danger or a Benefit for Atopics ? Curr Probl Dermatol $1999 ; 28: 81-7$.

4. AUMAITRE LA. Les aliments issus de plantes génétiquement modifiées : équivalence, efficacité et sécurité chez les animaux de ferme. INRA Prod Anim 2002 ; 15 : 97-108.

5. KUIPER HA, NOTEBORN HPJM, KOK EJ, KLETER GA. Safety aspects of novel foods. Food Research International $2002 ; 35: 267-71$.

6. SCALLA R. Biotransformation des herbicides par les plantes transgéniques résistantes. OCL 1997 ; $4: 113-9$.

7. BARLOW S, DYBING E, EDLER L, EISENBRAND G, KROES R, van den BRANDT P. Food Safety in Europe (FOSIE) : Risk Assessment of Chemicals in Food and Diet. Food and Chemical Toxicology 2002 ; $40(2 / 3): 137-427$.

8. CASTLE AL, CARVER MP, MENDRICK DL. Toxicogenomics : a new revolution in drug safety. Drug Discov Today $2002 ; 7$ : 728-36.

9. PARIS A. Profils suspects sous surveillance. La Recherche 2001 ; 339 : 74-7.

10. DUMAS ME, DEBRAUWER L, BEYET L, LESAGE D, ANDRE F, PARIS A, TABET JC. Analysing the physiological signature of anabolic steroids in cattle urine using pyrolisis/metastable atom bombardment mass sprectrometry and pattern recognition. Anal Chem 2002 ; 74 : 5393-404.

11. KEMPF H. L'expertise confidentielle sur un inquiétant maïs transgénique et Les effets à terme des OGM sont difficiles à mesurer, Le Monde 22/04/2004, Paris.

12. Libération des 8 et 9 mai 2004. 